\title{
The Impact of Gene-Environment Dependence and Misclassification in Genetic Association Studies Incorporating Gene-Environment Interactions
}

\author{
Sara Lindström ${ }^{a, b}$ Yu-Chun Yen ${ }^{a, b}$ Donna Spiegelman ${ }^{b, c}$ Peter Kraft ${ }^{a-c}$ \\ a Program in Molecular and Genetic Epidemiology, ${ }^{b}$ Department of Epidemiology, and \\ 'Department of Biostatistics, Harvard School of Public Health, Boston, Mass., USA
}

\section{Key Words}

Gene-environment interaction - Misclassification - Power •

Gene-environment dependence $\cdot$ Case control study

\begin{abstract}
The possibility of gene-environment interaction can be exploited to identify genetic variants associated with disease using a joint test of genetic main effect and gene-environment interaction. We consider how exposure misclassification and dependence between the true exposure $E$ and the tested genetic variant $G$ affect this joint test in absolute terms and relative to three other tests: the marginal test $(G)$, the standard test for multiplicative gene-environment interaction (GE), and the case-only test for interaction (GE-CO). All tests can have inflated Type I error rate when $E$ and $G$ are correlated in the underlying population. For the GE and G-GE tests this inflation is only noticeable when the gene-environment dependence is unusually strong; the inflation can be large for the GE-CO test even for modest correlation. The joint G-GE test has greater power than the GE test generally, and greater power than the $G$ test when there is no genetic main effect and the measurement error is small to moderate. The joint G-GE test is an attractive test for assessing genetic association when there is limited knowledge about casual mechanisms a priori, even in the presence of misclassification in environmental exposure measurement and correlation between exposure and genetic variants.
\end{abstract}

Copyright $\odot 2009$ S. Karger AG, Basel

\section{Introduction}

There is an increasing interest in leveraging the possibility of gene-environment interaction in order to identify genetic susceptibility loci in complex diseases. Considering that a genetic variant may only affect disease risk among those who are exposed to a particular environmental factor, incorporating gene-environment interaction in the analysis can provide more powerful tests of the null hypothesis that a locus is not associated with disease risk in any subpopulation. Allowing for gene-environment interaction can also provide stratum-specific estimates of the genetic effect, which may be more relevant for clinical and public health applications than population-averaged effects [1].

However, the standard, logistic-regression-based 'test for gene-environment interaction' suffers from several drawbacks. First, it is not sensitive to genetic main effects, which may be a drawback in the context of large-scale screening studies such as genome-wide association studies, where one is typically primarily interested in testing the global null that a genetic variant is not associated with disease risk in any stratum, regardless of whether the effect is constant across strata or not. Second, because the standard test is specifically a test for departures from a multiplicative odds ratio model for the joint effect of a genetic variant and an environmental exposure, the test is scale-dependent: additivity (i.e. 'no interaction') on the log-odds scale almost always implies non-additivity ('in-

\section{KARGER}

Fax +4161306 1234 E-Mail karger@karger.ch www.karger.com
(C) 2009 S. Karger AG, Basel

0001-5652/09/0683-0171\$26.00/0

Accessible online at:

www.karger.com/hhe
Sara Lindström

Department of Epidemiology

Harvard School of Public Health, Building 2, Room 211A

655 Huntington Avenue, Boston, MA (USA)

Tel. +1 617432 5896, Fax +1 617432 1722, E-Mail slindstr@hsph.harvard.edu 
teraction') on the absolute risk scale, for example. This makes the test difficult to interpret [2-4]. Finally, the standard test for interaction is notoriously data-hungry: assuming interaction effects are on the order of what has been observed for genetic main effects discovered via genome-wide association studies or a little larger (odds ratios from 1.2 to 2.0 ), more than 10,000 cases-control pairs will be needed to reliably detect 'gene-environment interactions' [5].

Recently, Kraft et al. [6] proposed a joint test of marginal genetic association and gene-environment interaction for case-control data (denoted as the G-GE test throughout the rest of this paper). This test exploits geneenvironment interaction to identify genetic associations by allowing for the possibility of a genetic effect to be modified by an environmental exposure E. Essentially, the test assesses the genetic main effect and the gene-environment interaction simultaneously through a likelihood ratio test with 2 degrees of freedom. The G-GE test is generally more powerful than the marginal genetic association test when the genetic effect is restricted to exposed subjects. In addition, it generally outperforms the conventional gene-environment interaction (GE) test (which assesses the interaction term only), especially when there is a genetic main effect. Even when the G-GE test is not the most powerful, the loss in power relative to the most powerful test is moderate. This makes the G-GE test a good choice when little is known a priori about variation in the strength of genetic association across exposure strata, as will be the case in studies that use anonymous tagging Single Nucleotide Polymorphisms (SNPs) to measure genetic variation across candidate regions or the entire genome.

Measurement error in exposure assessment is a major source of bias and loss of power in epidemiological studies. The impact of misclassification in gene-environment interaction studies as well as methods for correcting for it have been discussed previously [7-10]. However, these papers all assume that the tested genetic variant is independent of the environmental exposure in the underlying population, which is not always the case [11].

We give two examples of gene-environment dependence. First, genetic variation may be causally related to exposure, especially behavioral or anthropometric characteristics (e.g. smoking patterns, body mass index). This is the case with SNPs in the CHRNA3/5 region, which have been associated with number of cigarettes smoked per day among ever smokers [12-15]. These SNPs are also associated with lung cancer $[16,17]$. Currently, it is not clear if these SNPs influence risk of lung cancer solely through their influence on smoking behavior, or if they also influence risk along another pathway $[18,19]$. Second, genetic variation and exposure may be correlated because they are both influenced by an unmeasured confounder. Socio-economic status, exposure to air pollution, and different dietary patterns are all correlated with race and ethnicity in the United States, which may lead to a non-causal correlation between these exposures and genetic variants whose frequency differs within and among ethnic groups $[20,21]$.

In this paper, we consider how the Type I error rates in gene-environment interaction studies are affected by assessment of a misclassified surrogate $X$ instead of the true exposure $E$, when $E$ is associated with disease and $G$ and $E$ are dependent in the underlying population. In addition, we examine how environmental exposure misclassification affects gene-environment interaction studies in terms of power.

More specifically, we compare four simple likelihood ratio tests: the marginal genetic $(\mathrm{G})$ test, the joint $\mathrm{G}-\mathrm{GE}$ test, the conventional gene-environment interaction test (GE) and case-only test for gene-environment interaction (GE-CO). We present calculations for Type I error rates and power for a wide range of scenarios varying allele and exposure frequency, baseline disease risk, $\alpha$-level (i.e. nominal Type I error rate), effect size, magnitude of geneenvironment correlation, and extent of exposure misclassification.

\section{Statistical Methods}

We present methods for unmatched case-control data, the extension to matched case-control and family-based designs is straightforward [22]. We denote disease status as $D$ (1 for affected, 0 for unaffected) and the binary environmental exposure as $E$ ( $E=0$ if unexposed and $E=1$ if exposed). We assume a dominant inheritance model for the risk allele $G(G=1$ for carriers of at least one risk allele and 0 otherwise). These calculations are easily modified to allow for other genetic models including recessive and multiplicative. We assume that the genotypes are in HardyWeinberg equilibrium (i.e. $\mathrm{P}(G)=p_{g}\left(2-p_{g}\right)$, where $p_{g}$ is the risk allele frequency). The true penetrance model of disease $D$ is assumed to be:

$$
\log \frac{P(D=1 \mid G, E)}{P(D=0 \mid G, E)}=b+b_{g} G+b_{e} E+b_{g e} G E,
$$

where $b_{g e}$ is the interaction parameter for $G$ and $E$, i.e. $\mathrm{OR}_{\mathrm{ge}}=$ $\exp \left(b_{g e}\right)$ is the ratio of the genetic odds ratio in subjects exposed to the environmental factor relative to individuals not exposed to the environmental factor (we use Roman letters to denote param- 
eters in the true penetrance model and Greek letters to denote parameters estimated from the data).

Note that although we have parameterized the true model so that $b_{g e}$ represents the departure from a multiplicative odds model for the joint effect of $G$ and $E$ (i.e. $b_{g e}$ quantifies the magnitude of the departure from an additive model on the log-odds scale, colloquially known as a 'multiplicative interaction'), this does not prevent us from estimating the power of these tests in the presence of a departure from an additive model on the absolute risk scale (colloquially known as an 'additive interaction') [23]. For example, consider the hypothesized true model where the risk in unexposed noncarriers is 1 per 10,000; the risk in exposed noncarriers is 3 per 10,000; in unexposed carriers is 1.5 per 10,000 ; and the risk in exposed carriers is 4.5 per 10,000 . This model represents a supra-additive interaction $(4.5>1+2+0.5)$, but an absence of multiplicative interaction $(4.5=3 \times 1.5)$. This model can be represented using $b_{g}=\log (1.5), b_{e}=\log (3)$, and $b_{g e}$ $=0$. (The intercept term $b$ depends on the allele frequency and prevalence of $E$ ).

If we assume that there is no dependence between $G$ and $E$ in the underlying population (i.e. $\mathrm{P}(G, E)=\mathrm{P}(G) \mathrm{P}(E)$ ), the population prevalence of disease is given by:

$$
K_{p}=\sum_{G, E} P(G) P(E) P(D=1 \mid G, E)
$$

When $G$ and $E$ are dependent in the underlying population we propose the following expression for the population prevalence of disease:

$$
K_{p}=\sum_{G, E} P(G) \frac{\exp \left(E\left(a_{e}+a_{g e} G\right)\right)}{1+\exp \left(a_{e}+a_{g e} G\right)} P(D=1 \mid G, E) .
$$

Here

$$
a_{e}=\log \left(\frac{p_{e}}{1-p_{e}}\right)
$$

( $p_{e}$ is the probability $E=1$ when $G=0$ ) and $a_{g e}$ parameterizes the gene-environment dependence ( $G$ and $E$ are independent for $a_{g e}=0$ and the strength of the dependence increases as $\left|a_{g e}\right|$ increases).

We now extend this model to incorporate an observed surrogate $X$ for the unobserved true exposure $E$. We assume that any misclassification in $X$ is non-differential, i.e. the misclassification error does not depend on disease status $D$. Moreover, we assume that conditional on $E$, the observed exposure $X$ is independent of $G$ (i.e. the probability any exposure $E$ is misclassified is constant over different genotypes); that conditional on $E$ disease risk is independent of the measured exposure $X$; and that sampling is independent of $G, X$ and $E$, conditional on $D$.

We specify the degree of misclassification through sensitivity and specificity parameters, where sensitivity is the probability that a truly exposed subject is classified as exposed and specificity as the probability that a truly non-exposed subject is classified as non-exposed. If we allow for gene-environment dependence in the underlying population, then the joint probability of the observed unmatched case-control data $f_{\mathbf{b}, \mathbf{s}, \mathbf{a}, p_{g}}(D, G, X)$ for a subject will depend on the degree of misclassification and gene-environment dependence:

$$
\begin{aligned}
& f_{\mathbf{b}, \mathbf{s}, \mathbf{a}, p_{g}}(D, G, X)= \\
& \frac{\sum_{X, G, E} P_{b, b_{g}, b_{e}, b_{g e}}(D \mid G, E) P_{s_{0}, s_{1}}(X \mid E) P_{a_{g e}, a_{e}}(E \mid G) P_{p_{g}}(G)}{K_{p}^{D}\left(1-K_{p}\right)^{1-D}} \pi(D) .
\end{aligned}
$$

This expression factors the joint probability of $D, G, X$ and $E$ into four terms. The first term is simply the probability of disease given genotype and true (unobserved) environmental exposure and depends on the penetrance parameters $b, b_{g}, b_{e}$ and $b_{g e}$; the second term expresses the relationship between $E$ and $X$ as a function of the sensitivity $s_{1}$ and specificity $s_{0}$; and the last two terms model the joint distribution of $G$ and $E$. The term $\pi(D)$ represents the marginal probability for case-control status $D$, conditional on selection into the case-control study. In other words, for $D=1, \pi(D)$ is the number of cases divided by the total number of subjects in the case-control study, and for $D=0, \pi(D)$ is the fraction of the total sample that is controls. Here we assume 1:1 case:control sampling, so $\pi(D) \equiv 1 / 2$.

We calculated type I error rates and power for four different likelihood ratio test statistics. These test statistics are approximately $\chi^{2}$ distributed, with non-centrality parameter $[22,24]$ :

$$
\delta=2 n \sum_{D, G, X}\left[l_{1}\left(\hat{\boldsymbol{\beta}}_{1} ; D, \mathrm{G}, X\right)-l_{0}\left(\hat{\boldsymbol{\beta}}_{0} ; D, \mathrm{G}, X\right)\right] f_{\mathbf{b}, \mathbf{s}, \mathbf{a}, p_{g}}(D, G, X) .
$$

Here $l_{1}$ and $l_{0}$ are the log-likelihoods of the observed data under the alternative and null hypotheses, and $\hat{\boldsymbol{\beta}}_{1}$ and $\hat{\boldsymbol{\beta}}_{0}$ are the values of the regression parameters that maximize the corresponding expected log likelihoods (where the expectation is taken over the distribution specified by $\boldsymbol{b}, \boldsymbol{s}, \boldsymbol{a}$ and $p_{g}$ ). The number of total subjects (cases plus controls) is denoted by $n$. We calculate $\delta$ using the exemplary data procedure as described in [24], implemented in a SAS macro (available at www.hsph.harvard.edu/faculty/kraft/ soft.html). The degrees of freedom for the distribution of the test statistic is given by $\operatorname{dim}\left(\hat{\boldsymbol{\beta}}_{1}\right)-\operatorname{dim}\left(\hat{\boldsymbol{\beta}}_{0}\right)$.

We compared type I error rates and power for the marginal $\mathrm{G}$ test, the joint G-GE test, the conventional gene-environmental GE test, and the case-only gene-environment interaction (GE$\mathrm{CO})$ test. The first three tests are based on standard logistic regression for case-control data but use different alternative and null log-likelihoods. The marginal $\mathrm{G}$ test compares the alternative likelihood $\exp \left(\beta+\beta_{g} G\right)^{D} /\left(1+\exp \left(\beta+\beta_{g} G\right)\right)$ to the null likelihood $\exp (\beta)^{D} /(1+\exp (\beta))$. This leads to a one degree of freedom test. The GE and G-GE tests compare the saturated alternative likelihood $\exp \left(\beta+\beta_{g} G+\beta_{e} E+\beta_{g e} G E\right)^{D} /\left(1+\exp \left(\beta+\beta_{g} G+\beta_{e} E\right.\right.$ $\left.\left.+\beta_{g e} G E\right)\right)$ to two different null likelihoods: $\exp \left(\beta+\beta_{g} G+\beta_{e} E\right)^{D} /$ $\left(1+\exp \left(\beta+\beta_{g} G+\beta_{e} E\right)\right)$ for the GE test and $\exp \left(\beta+\beta_{e} E\right)^{D} /$ $\left(1+\exp \left(\beta+\beta_{e} E\right)\right)$ for the G-GE test. These tests have one and two degrees of freedom, respectively.

Note that just as we were able to represent any pattern of geneenvironment interaction using the saturated parameterization of model [1], the joint test is sensitive to a wide range of gene-environment interaction patterns. This is different than the standard test for interaction, which only tests $\beta_{g e}=0$ and hence can only detect departures from additivity on the log odds scale ('multiplicative interaction'). In fact, the joint test is scale free, in the sense that any parameterization of the null model (two levels of risk, depending on exposure) and the alternative (four levels of risk, depending on genetic variant and exposure) would lead to the same likelihood ratio test. In particular, parameterizing in terms 
of risk differences - i.e. $P(D=1 \mid G, E)=\beta^{*}+\beta_{g}^{*} G+\beta_{e}^{*} E+\beta_{g e}^{*} G E$ for the saturated model - yields the same joint test as the logistic parameterization (although the tests of $\beta_{g e}=0$ and $\beta_{g e}^{*}=0$ are generally different).

The case-only test is equivalent to a logistic regression of $X$ on $G$ among cases. We calculated the noncentrality parameter for this test using a modification of the exemplary data procedure described above: taking the expectation of a likelihood ratio over the true distribution of $G$ and $X$ among cases.

We explored Type I error and power in different scenarios by varying several parameters including: baseline disease prevalence $\in(0.01,0.0001)$; main effect of environmental exposure $\left.\mathrm{OR}_{\mathrm{e}}=\exp \left(b_{e}\right)\right) \in(1.2,1.6,2.0,2.4)$ environmental exposure frequency in non-carriers $p_{e} \in(0.1,0.25,0.5,0.75)$; risk allele frequency $p_{g} \in(0.05,0.1,0.25,0.4)$ and gene-environment dependence odds ratios $\exp \left(a_{g e}\right) \in(1.0,1.2, \ldots, 3.0)$. Note that we assume throughout the calculations that $E$ is associated with disease in all strata defined by $G$, as we are primarily motivated by the case where $E$ is a known risk factor. We also varied sensitivity and specificity in the ranges $(0.2,0.3, \ldots, 0.9)$ and $(0.3,0.4, \ldots, 0.9999)$, respectively. Specificity was constrained to be larger than sensitivity, which is typical of most surrogate exposures used in epidemiologic studies. We restricted our attention to situations where $X$ is a misclassified measure of $E$, as the situation where $E$ is measured without error has been considered previously [25]. We varied nominal Type I error levels: $\alpha \in(0.05,0.01,0.001,0.0001)$. For power calculations, we considered a genetic main effect of 1.2 , environmental main effect of 1.5 and gene-environment interaction effects of 1.2, 1.6 and 2.0. For both power and Type I error rate calculations, we considered studies with 600 cases and 600 controls. In total, we calculated Type I error or power in 253,440 different scenarios.

\section{Results}

In general, baseline disease prevalence had no impact on the results, and we observed the same relative patterns for different $\alpha$-levels even though the absolute magnitude of the power and type I error rates depended on the nominal $\alpha$ level. All of the results presented in this section assume $\alpha=0.01$ and baseline disease prevalence $=0.0001$; complete results for all of the scenarios we considered are available at (www.hsph.harvard.edu/faculty/kraft/soft. html).

\section{Type I Error Rates}

Table 1 shows the type I error rates for a subset of the values of sensitivity, specificity and gene-environment dependence we considered. We present results for sensitivity ranging between 0.5 and 0.9 , allele frequency $p_{g}=$ 0.25 , exposure frequency $p_{e}=0.25$, and exposure odds ratio $\mathrm{OR}_{\mathrm{e}}=1.6$.

All four tests had valid Type I error rates when $E$ and $G$ were independent in the general population $\left(a_{g e}=0\right)$, even when sensitivity and specificity were less than one. When $E$ and $G$ are dependent in the underlying population $\left(a_{g e} \neq 0\right)$ and $X$ is a misclassified measure of $E$ the GE test generally had the least inflation in Type I error rates in nearly all scenarios considered, followed by the G-GE test, the $\mathrm{G}$ test, and then the GE-CO test with the highest inflation in Type I error rates. (It has been previously shown that when $E$ is measured without error, but $G$ and $E$ are correlated in the general population, the GE and G-GE tests have proper size, while the GE-CO and G tests can have inflated Type I error rates [6]). We observed departures from this ranking only in occasional scenarios. For example, the GE test had largest inflation in Type I error rates in $0.01 \%$ of the scenarios considered and this occurred under extreme parameter values given by $p_{g} \geq$ $0.25, p_{e}=0.75, \mathrm{OR}_{\mathrm{e}}=2.4$, sensitivity $=0.8$, specificity $=$ 0.9999 and $\exp \left(a_{g e}\right)>2.4$. Similarly, the G-GE test had most inflated Type I error rate $0.04 \%$ of the time and this was only when $p_{g} \geq 0.25, p_{e}=0.75, \mathrm{OR}_{\mathrm{e}} \geq 2.0$, sensitivity $\geq 0.6$, specificity $=0.9999$ and $\exp \left(a_{g e}\right) \geq 2.4$. However, except from GE-CO test which always exhibits much higher Type I error rates, the differences in Type I errors were very small in these cases that deviated from the general pattern.

The G test had highly inflated Type I error rates when the gene-environment independence assumption was violated. This is because the association between the genetic variant and disease is confounded by the exposure, and the marginal test makes no attempt to adjust for this confounding. (The estimate $\beta_{g}$ does not converge to the true penetrance parameter $b_{g}$ ). We noticed an almost 3fold increase in type I error rate when $\exp \left(a_{g e}\right)=2$, which corresponds to a very strong association between the exposure and the risk genotype. The type I error rate for the $G$ test is reduced as the environmental effect, the allele frequency or the correlation between the genetic variant and exposure decreases.

The GE test is robust to gene-environment dependence and shows only slightly increased Type I error rates when the dependence is strong $\left(\exp \left(a_{g e}\right)>2\right)$ and the environmental exposure frequency and effect is high. The G-GE test is sensitive to misclassification and becomes more inflated as gene-environment dependence, misclassification error, allele frequency and the size of the environmental effect increases. This is because the main effect of the genetic variant is confounded by residual variation in $E$, conditional on $X$ [26]. However, the inflation of the Type 1 error is only noteworthy when the gene-environment interaction is strong, misclassification is prominent (specificity and 
Table1. Type I error rates for four different tests (G-GE, G, GE, GE-CO; see Methods) for different combinations of sensitivity, specificity and GE correlation in the underlying population. Based on a study with 600 cases and 600 controls where a dominant inheritance was assumed

\begin{tabular}{|c|c|c|c|c|c|c|c|c|c|c|c|c|c|}
\hline Sens & Spec & $\theta_{\mathrm{ge}}$ & G-GE & G & GE & GE-CO & Sens & Spec & $\theta$ ge & G-GE & G & GE & GE-CO \\
\hline \multirow[t]{30}{*}{0.5} & \multirow[t]{6}{*}{0.6} & 1.0 & 0.010 & 0.010 & 0.010 & 0.010 & \multirow[t]{9}{*}{0.6} & \multirow[t]{3}{*}{0.9} & 1.6 & 0.012 & 0.017 & 0.010 & 0.140 \\
\hline & & 1.2 & 0.011 & 0.011 & 0.010 & 0.010 & & & 1.8 & 0.014 & 0.022 & 0.010 & 0.242 \\
\hline & & 1.4 & 0.012 & 0.013 & 0.010 & 0.011 & & & 2.0 & 0.015 & 0.028 & 0.010 & 0.358 \\
\hline & & 1.6 & 0.014 & 0.017 & 0.010 & 0.013 & & \multirow[t]{6}{*}{0.999} & 1.0 & 0.010 & 0.010 & 0.010 & 0.010 \\
\hline & & 1.8 & 0.017 & 0.022 & 0.010 & 0.015 & & & 1.2 & 0.010 & 0.011 & 0.010 & 0.034 \\
\hline & & 2.0 & 0.021 & 0.028 & 0.010 & 0.017 & & & 1.4 & 0.011 & 0.013 & 0.010 & 0.116 \\
\hline & \multirow[t]{6}{*}{0.7} & 1.0 & 0.010 & 0.010 & 0.010 & 0.010 & & & 1.6 & 0.011 & 0.017 & 0.010 & 0.258 \\
\hline & & 1.2 & 0.011 & 0.011 & 0.010 & 0.012 & & & 1.8 & 0.012 & 0.022 & 0.011 & 0.432 \\
\hline & & 1.4 & 0.012 & 0.013 & 0.010 & 0.016 & & & 2.0 & 0.014 & 0.028 & 0.011 & 0.598 \\
\hline & & 1.6 & 0.014 & 0.017 & 0.010 & 0.023 & \multirow[t]{18}{*}{0.7} & \multirow[t]{6}{*}{0.8} & 1.0 & 0.010 & 0.010 & 0.010 & 0.010 \\
\hline & & 1.8 & 0.017 & 0.022 & 0.010 & 0.032 & & & 1.2 & 0.010 & 0.011 & 0.010 & 0.021 \\
\hline & & 2.0 & 0.020 & 0.028 & 0.010 & 0.042 & & & 1.4 & 0.011 & 0.013 & 0.010 & 0.057 \\
\hline & \multirow[t]{6}{*}{0.8} & 1.0 & 0.010 & 0.010 & 0.010 & 0.010 & & & 1.6 & 0.013 & 0.017 & 0.010 & 0.120 \\
\hline & & 1.2 & 0.010 & 0.011 & 0.010 & 0.014 & & & 1.8 & 0.014 & 0.022 & 0.010 & 0.208 \\
\hline & & 1.4 & 0.012 & 0.013 & 0.010 & 0.026 & & & 2.0 & 0.016 & 0.028 & 0.010 & 0.312 \\
\hline & & 1.6 & 0.014 & 0.017 & 0.010 & 0.045 & & \multirow[t]{6}{*}{0.9} & 1.0 & 0.010 & 0.010 & 0.010 & 0.01 \\
\hline & & 1.8 & 0.016 & 0.022 & 0.010 & 0.071 & & & 1.2 & 0.010 & 0.011 & 0.010 & 0.029 \\
\hline & & 2.0 & 0.019 & 0.028 & 0.010 & 0.103 & & & 1.4 & 0.011 & 0.013 & 0.010 & 0.090 \\
\hline & \multirow[t]{6}{*}{0.9} & 1.0 & 0.010 & 0.010 & 0.010 & 0.010 & & & 1.6 & 0.012 & 0.017 & 0.010 & 0.201 \\
\hline & & 1.2 & 0.010 & 0.011 & 0.010 & 0.019 & & & 1.8 & 0.013 & 0.022 & 0.010 & 0.346 \\
\hline & & 1.4 & 0.011 & 0.013 & 0.010 & 0.046 & & & 2.0 & 0.014 & 0.028 & 0.010 & 0.499 \\
\hline & & 1.6 & 0.013 & 0.017 & 0.010 & 0.093 & & 0.999 & 1.0 & 0.010 & 0.010 & 0.010 & 0.010 \\
\hline & & 1.8 & 0.015 & 0.022 & 0.010 & 0.157 & & & 1.2 & 0.010 & 0.011 & 0.010 & 0.040 \\
\hline & & 2.0 & 0.017 & 0.028 & 0.010 & 0.233 & & & 1.4 & 0.010 & 0.013 & 0.010 & 0.148 \\
\hline & \multirow[t]{6}{*}{0.999} & 1.0 & 0.010 & 0.010 & 0.010 & 0.010 & & & 1.6 & 0.011 & 0.017 & 0.010 & 0.331 \\
\hline & & 1.2 & 0.010 & 0.011 & 0.010 & 0.029 & & & 1.8 & 0.012 & 0.022 & 0.011 & 0.538 \\
\hline & & 1.4 & 0.011 & 0.013 & 0.010 & 0.089 & & & 2.0 & 0.012 & 0.028 & 0.011 & 0.714 \\
\hline & & 1.6 & 0.012 & 0.017 & 0.011 & 0.194 & \multirow[t]{12}{*}{0.8} & \multirow[t]{6}{*}{0.9} & 1.0 & 0.010 & 0.010 & 0.010 & 0.010 \\
\hline & & 1.8 & 0.013 & 0.022 & 0.011 & 0.330 & & & 1.2 & 0.010 & 0.011 & 0.010 & 0.035 \\
\hline & & 2.0 & 0.015 & 0.028 & 0.011 & 0.472 & & & 1.4 & 0.011 & 0.013 & 0.010 & 0.121 \\
\hline \multirow[t]{15}{*}{0.6} & \multirow[t]{6}{*}{0.7} & 1.0 & 0.010 & 0.010 & 0.010 & 0.010 & & & 1.6 & 0.011 & 0.017 & 0.010 & 0.275 \\
\hline & & 1.2 & 0.011 & 0.011 & 0.010 & 0.014 & & & 1.8 & 0.012 & 0.022 & 0.010 & 0.464 \\
\hline & & 1.4 & 0.012 & 0.013 & 0.010 & 0.024 & & & 2.0 & 0.013 & 0.028 & 0.010 & 0.641 \\
\hline & & 1.6 & 0.014 & 0.017 & 0.010 & 0.041 & & \multirow[t]{6}{*}{0.999} & 1.0 & 0.010 & 0.010 & 0.010 & 0.010 \\
\hline & & 1.8 & 0.016 & 0.022 & 0.010 & 0.064 & & & 1.2 & 0.010 & 0.011 & 0.010 & 0.048 \\
\hline & & 2.0 & 0.019 & 0.028 & 0.010 & 0.092 & & & 1.4 & 0.010 & 0.013 & 0.010 & 0.185 \\
\hline & \multirow[t]{6}{*}{0.8} & 1.0 & 0.010 & 0.010 & 0.010 & 0.010 & & & 1.6 & 0.010 & 0.017 & 0.010 & 0.412 \\
\hline & & 1.2 & 0.010 & 0.011 & 0.010 & 0.017 & & & 1.8 & 0.011 & 0.022 & 0.010 & 0.643 \\
\hline & & 1.4 & 0.012 & 0.013 & 0.010 & 0.039 & & & 2.0 & 0.011 & 0.028 & 0.011 & 0.814 \\
\hline & & 1.6 & 0.013 & 0.017 & 0.010 & 0.076 & \multirow[t]{6}{*}{0.9} & 0.999 & 1.0 & 0.010 & 0.010 & 0.010 & 0.010 \\
\hline & & 1.8 & 0.015 & 0.022 & 0.010 & 0.128 & & & 1.2 & 0.010 & 0.011 & 0.010 & 0.056 \\
\hline & & 2.0 & 0.018 & 0.028 & 0.010 & 0.191 & & & 1.4 & 0.010 & 0.013 & 0.010 & 0.229 \\
\hline & 0.9 & 1.0 & 0.010 & 0.010 & 0.010 & 0.010 & & & 1.6 & 0.010 & 0.017 & 0.010 & 0.499 \\
\hline & & 1.2 & 0.010 & 0.011 & 0.010 & 0.023 & & & 1.8 & 0.010 & 0.022 & 0.010 & 0.742 \\
\hline & & 1.4 & 0.011 & 0.013 & 0.010 & 0.066 & & & 2.0 & 0.010 & 0.028 & 0.010 & 0.890 \\
\hline
\end{tabular}

Allele frequency and exposure frequency are 0.25 , environmental effect $\mathrm{OR}_{\mathrm{e}}$ is 1.6 , baseline disease prevalence is 0.0001 and $\alpha=$ 0.01. $\theta_{\mathrm{ge}}=\exp \left(a_{g e}\right)$ is the odds ratio for gene-environment correlation in the underlying population (see Methods). 
Fig. 1. Type I error rates for four different tests as a function of gene-environment odds ratio $\theta_{\mathrm{GE}}=\exp \left(a_{g e}\right)$ for different allele frequencies $p_{g}$ and exposure frequencies $p_{e}$. Based on a study with 600 cases and 600 controls where a dominant inheritance was assumed. Sensitivity is 0.6 , specificity is 0.9 , environmental effect $\mathrm{OR}_{\mathrm{e}}$ is 1.6 , baseline disease prevalence is 0.0001 and $\alpha=0.01$

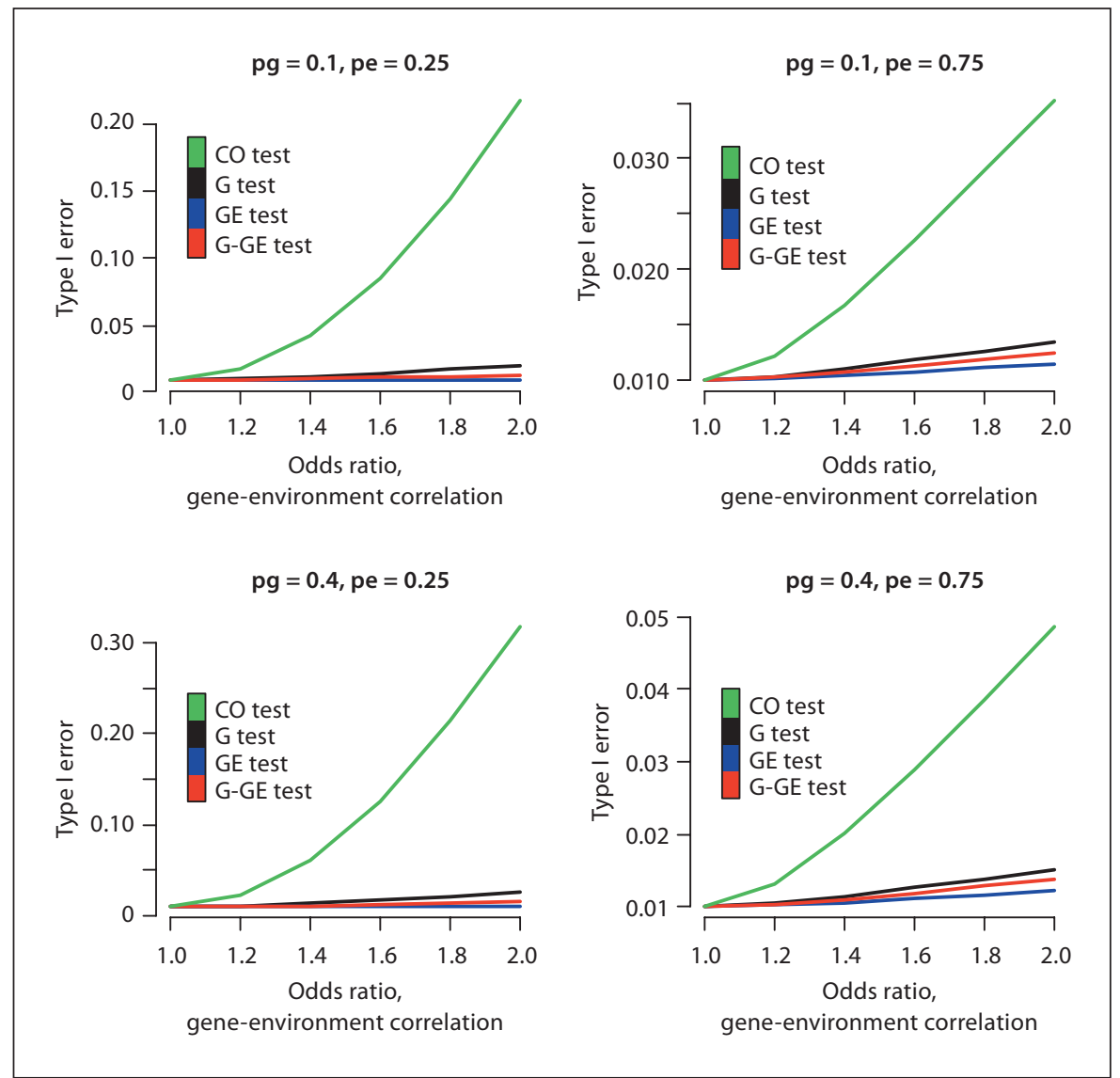

sensitivity <0.7) and the environmental effect is high $\left(\mathrm{OR}_{\mathrm{e}} \geq 1.6\right)$ [27].

Figure 1 illustrates the Type I error as a function of dependence between $G$ and $E$ in the underlying population for four different combinations of allele and exposure frequency. As can be seen, the GE-CO test followed by the $\mathrm{G}$ test has the most inflated Type I error rates. The inflation is most prominent when the exposure frequency is low and allele frequency is high. The GE test is fairly robust to exposure frequency but shows a slightly inflated type I error when $p_{e}=0.75$ while the G-GE test seems most affected by a low exposure frequency.

It is well-known that the case-only test is powerful in assessing gene-environment interaction as long as $G$ and $E$ are independent in the study base. However, if this assumption is not true, the test will be biased. We assessed the magnitude of this error performing the same calculations described above. As gene-environment dependence increases, the case-only test is by far the most biased test of those included. This is true especially if the misclassification is low, which is not surprising given that the case-only test measures the association between $G$ and $E$, with $E$ as the outcome. For example, when allele frequency is 0.25 , exposure frequency $p_{e}=0.25$, exposure odds ratio $\mathrm{OR}_{\mathrm{e}}=1.6$, sensitivity $=0.7$, specificity $=0.8$ and $\exp \left(a_{g e}\right)=1.6$, the type I error rate for the case-only test is 0.120 compared to 0.014 for the G-GE test, 0.022 for the $\mathrm{G}$ test and 0.010 for the GE test. In general, sensitivity and G-E dependence are the two most influential parameters on the type I error for the case-only test.

In general, negative correlation between the risk genotype $G$ and deleterious exposure $E$ did not alter these observations regarding the Type I error rate (results not shown). The GE-CO test has most inflated type I error rates while the GE test suffers the least. In addition, the magnitude of the inflation is similar as with positive correlation.

\section{Power}

We restrict our discussion of power to situations where $G$ and $E$ are independent in the general population. In these situations, all four tests will have valid Type I error 


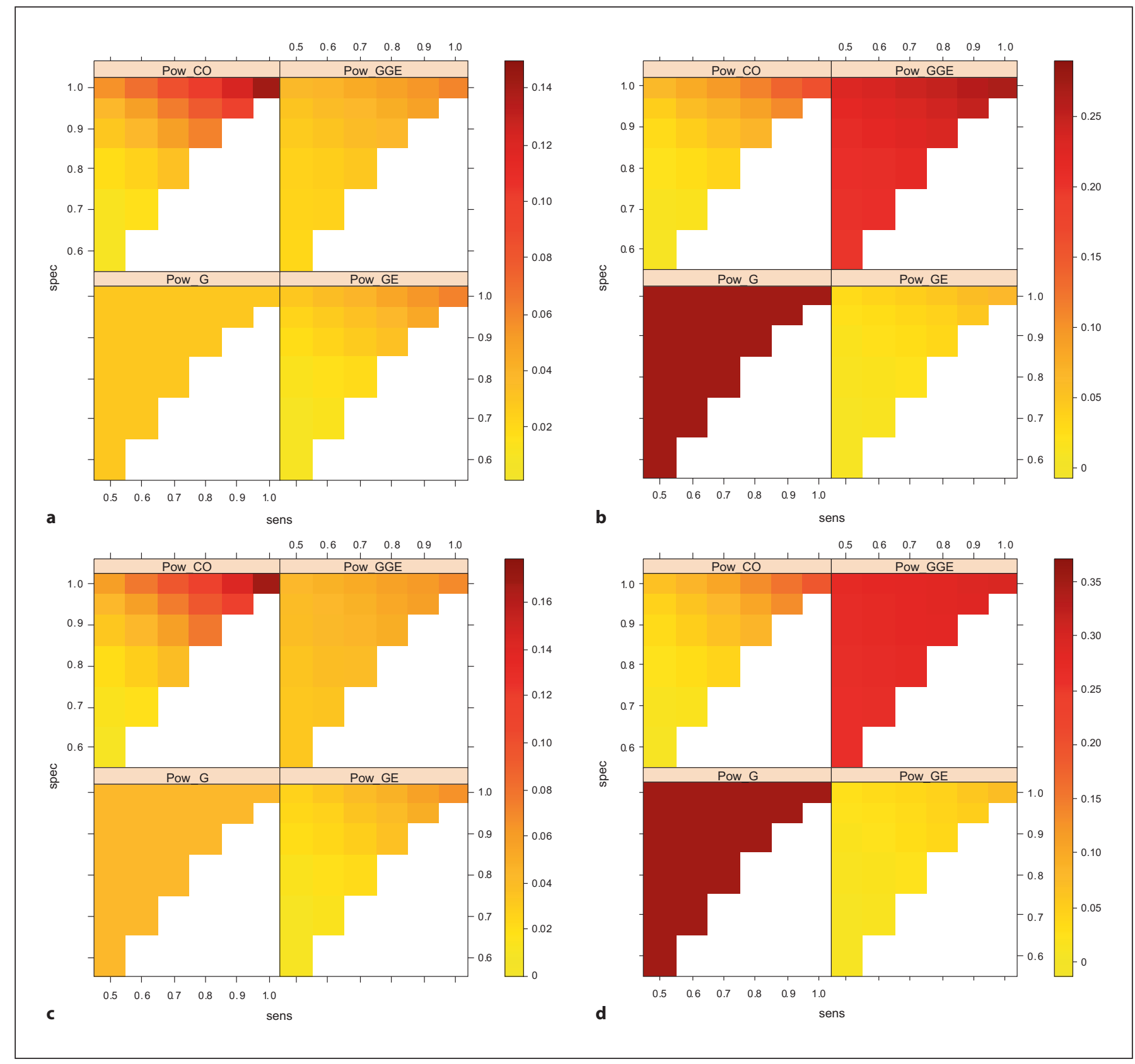

Fig. 2. Power for four different tests as a function of sensitivity and specificity for different genetic and gene-environment interaction effects. Based on a study with 600 cases and 600 controls where a dominant inheritance was assumed. The allele frequency $p_{g}$ is 0.1 and the environmental frequency $p_{e}$ is 0.25 . The gene-environment interaction effect $\mathrm{OR}_{\mathrm{ge}}$ is 1.4, baseline disease prevalence is 0.0001 and $\alpha=0.01 . \mathbf{a} \mathrm{OR}_{\mathrm{g}}=1.0, \mathrm{OR}_{\mathrm{e}}=1.0 . \mathbf{b} \mathrm{OR}_{\mathrm{g}}=1.2, \mathrm{OR}_{\mathrm{e}}=$ 1.0. $\mathrm{c} \mathrm{OR}_{\mathrm{g}}=1.0, \mathrm{OR}_{\mathrm{e}}=1.5 . \mathbf{d} \mathrm{OR}_{\mathrm{g}}=1.2, \mathrm{OR}_{\mathrm{e}}=1.5$. rates. Figure 2 illustrates the power for the four tests as a function of sensitivity and specificity, for four different true penetrance models (with different genetic and environmental main effects). Consistent with previous results for non-misclassified $X$, the joint test has greater power than the G test when the genetic main effect $b_{G}=0$ and there is little misclassification error (sensitivity and specificity are high). As misclassification error increases, the power of the G-GE test declines and can be lower than that of the $G$ test (e.g. when sensitivity $\leq 0.6$ and specific- 
ity $\leq 0.8$ in fig. $2 a)$. The $G$ test is naturally robust to exposure misclassification (as it does not use data on $X$ ). The GE test almost always performs worse than the $G$ and G-GE test in terms of power regardless of the misclassification error, most noticeably so when there is a genetic main effect (fig. 2b, c). This is also in agreement with earlier findings [6]. When there is no genetic main effect and small to moderate misclassification, the GE-CO test is most powerful although power decreases rapidly as misclassification increases. When there is a genetic main effect, the GE-CO test is more powerful than the GE test but can have much less power than both the $G$ and the G-GE test, consistent with previous observations [6].

To further illustrate how misclassification affects the power of these tests, we performed power calculations using sensitivity and specificity derived from a real-data example. Sensitivity and specificity parameters for body mass index (BMI) were estimated using 7,455 participants where both self-reported and measured height and weight were available [28]. Obesity was defined by having a BMI $>30 \mathrm{~kg} / \mathrm{m}^{2}$. Overall, sensitivity was estimated to $74 \%$ and specificity $99 \%$ and $15 \%$ of the participants were classified as obese using measured BMI. Assuming a study of 600 cases and 600 controls, an allele frequency of 0.25 , genetic main effect of 1.2 , interaction effect of 1.6 and an environmental effect of 1.5 , the power to detect an association at a $1 \%$ significance level is 0.45 for the G-GE test, 0.51 for the G test and 0.09 for the GE test. If we assume no genetic main effect the corresponding power is 0.10 for the G-GE test, 0.06 for the G test and 0.09 for the GE test, respectively.

Assuming no misclassification, the power to detect an association was 0.47 for the G-GE test, 0.51 for the G test and 0.15 for the GE test. In the absence of a genetic main effect, the power is 0.14 for the G-GE test, 0.06 for the G test and 0.16 for the GE test. This is consistent with the results given in figure 2: the $G$ test maintains its power despite misclassification while the G-GE test suffers from a small loss in power.

\section{Discussion}

In this paper, we examined how misclassification and gene-environment dependence can affect the validity of four commonly used tests for detecting a genetic association in the presence of gene-environment interaction. If $G$ and $E$ are independent in the underlying population, then all four tests remain valid tests for variant-disease association despite exposure misclassification, although there can be a loss of power, whose magnitude depends on the sensitivity and specificity of $X$ for $E$. However, if $G$ and $E$ are dependent in the study base, then all four tests can have inflated Type I error rates, and the degree of inflation depends on the strength of the gene-environment correlation and the sensitivity and specificity of $X$ for $E$. We observed highly inflated Type I error rates for the genetic main effect $\mathrm{G}$ test and the case-only test for interaction GE-CO when the gene-environment independence assumption is violated, regardless of degree of misclassification. The GE test, on the other hand, is relatively robust to gene-environment dependence and only shows higher Type I error rates when the environmental effect is high and there is a strong gene-environment correlation.

Assuming gene-environment independence, we compared the four tests in terms of power in the presence of misclassification of the environmental exposure. We observed a strong relationship between the magnitude of the misclassification and power for the G-GE test, where statistical power drops when sensitivity and specificity goes down. The power of the GE and GE-CO test was also strongly reduced by misclassification and had a greater proportionate loss of power than the G-GE test. We also showed that the G-GE test outperformed the G test when no genetic main effect was present and exposure misclassification was moderate.

Like the marginal G test, the joint G-GE test is a test of the global null hypothesis that the genetic variant is not associated with disease risk in any stratum defined by exposure (i.e. $b_{g}=b_{g e}=0$ in terms of model [1]). This makes it appropriate for studies whose primary goal is discovering new disease loci, e.g. genome-wide association scans. Because the joint test allows the genetic effect to vary across exposure strata, it can be more powerful than the marginal test in situations where there are known environmental risk factors. By using a saturated parameterization, the joint test is sensitive to a range of alternatives consistent with different interaction patterns (e.g. 'additive' or 'multiplicative' interactions). However, while this scale-free property is a strength in the context of discovery, it is a drawback in the context of characterizing a variant already known to be associated with disease risk, that is, specifically asking whether or not the pattern of risks is consistent with a particular model for the joint effects of the genetic variant and exposure, in order to draw conclusions about etiology or public health impact. For example, in the sufficient component cause or counterfactual frameworks, departures from additivity on the absolute scale $\left(b_{g e}^{*} \neq 0\right.$ in the notation used in 
the Methods section) imply that there exist some individuals whose disease risk depends on both their genotype and their exposure profile [23]. Although more commonly used (because of mathematical convenience), tests for departures from additivity on the log odds scale $\left(b_{g e} \neq 0\right)$ generally require strong (untestable) assumptions to be interpreted etiologically $[23,29]$ Regardless of scale, the joint test is a poor test of interaction per se, as by construction it is sensitive to both genetic main effects and gene-environment interactions.

The case-only test is often proposed as the most powerful test to detect ('multiplicative') gene-environment interaction. Although this is true in many situations, the case-only test assumes gene-environment independence and is therefore biased when this assumption is violated. Thus, the case-only test should be used with care [30]. In a recent paper, Mukherjee and colleagues proposed a novel empirical Bayes method to test for gene-environment interaction [31]. This approach weights the parameter estimates from a standard analysis and an alternative constrained analysis that assumes gene-environment independence (analogous to the case-only test) according to the evidence against the hypothesis of gene-environment independence. They compared their test with three others including the GE test and concluded that their test and the GE test are both robust to gene-environment dependence. They also noted that their test had more power than the others when the gene-environment independence assumption was fulfilled [25]. However, they did not consider misclassification or the G-GE test when comparing different tests in terms of Type I error rates and power. The performance of this test in the presence of exposure misclassification warrants further investigation.

Measurement error is inevitable in observational epidemiological studies. Ignoring misclassification error may result in biased point estimates and invalid measures of association. Although there is a wide awareness of misclassification in exposure assessment and methods for study design and data analyses which avoid the bias have long been available [32-35], it is rarely remedied in the final analysis, leading to inconclusiveness and spurious findings. For gene-environment interaction studies, optimal main study/validation study designs in the presence of exposure misclassification have been studied to some extent [10], but require further attention. Wong and colleagues showed that it is sometimes more efficient to conduct smaller studies with more precise exposure and outcome measurements [36] than to include a large number of subjects in the study. Power calculations such as those presented here can help investigators interested in gene-environment interactions choose between designs that measure exposure extremely accurately on a small number of subjects and designs that measure exposure somewhat less accurately, but on a large number of subjects.

Gene-environment dependence in the underlying population has often been discussed in epidemiology. This dependence could arise for a number of reasons. A genetic variant and exposure could be correlated due to population stratification, for example, if allele frequencies and exposure frequencies are correlated across latent subpopulations (even though genotypes and exposure are independent within the subpopulations). Gene-environment dependence could also arise if the genetic variant influences behaviour, which in turn affects exposure probability. A recently discovered example of this is the association between the CHRNA3 and CHRNA5 genes and number of cigarettes smoked per day among smokers [12-15]. The calculations we presented here make no assumptions about the source of the gene-environment dependence (e.g. whether $G$ causes $E$ or $G$ and $E$ share an unmeasured cause), but we urge care in thinking through the implications of different mechanisms for gene-environment dependence when choosing and interpreting an analysis. For example, if $G$ causes $E$, then the marginal $G$ test will reject the null hypothesis at higher rate than the nominal Type I error rate - but depending on the context, this may be interesting and useful. If one did not know that variants in the CHRNA3/5 region were associated with lifetime exposure to cigarette smoking, then a marginal association between genetic variants in this region and lung cancer (as has been observed $[16,17]$ ) might lead to new insights - in particular, ancillary analyses might uncover the link between this region and smoking behaviour. On the other hand, if one knew of the CHRNA3/5smoking link and wished to test whether variants in this region cause lung cancer independent of their association with smoking (as has been hypothesized [18]), then the marginal test is not useful. Similarly, if one is concerned about confounding due to unmeasured common causes of the genetic variation and exposure (as in the population stratification example), then the marginal $G$ test (and to a lesser extent the G-GE test in the presence of exposure misclassification) may be confounded.

This paper is, to our knowledge, the first that makes a simultaneous assessment of GE dependence and misclassification error and how they affect test validity and power for gene-environment association tests. We have shown that the joint G-GE test is almost always less biased than 
the $\mathrm{G}$ test when $G$ and $E$ are correlated in the underlying population. Although the G-GE test is biased in the presence of gene-environment correlation, the inflation of the Type I error rate only becomes noteworthy when the gene-environment dependence is unusually strong. We have also shown in this paper that the joint G-GE test outperforms the conventional GE test in terms of power when misclassification is present, and it outperforms the marginal G test when there is no genetic main effect and the measurement error is small to moderate. These re- sults make the joint G-GE test an attractive test for assessing gene-environment interactions in genetic epidemiology when there is limited knowledge about casual mechanisms a priori.

\section{Acknowledgements}

This work was supported by NCI grant 5R01CA50597-15. SL was supported by the Swedish Research Council.

\section{References}

1 Hunter DJ: Gene-environment interactions in human diseases. Nat Rev Genet 2005;6: 287-298.

2 Chatterjee N, Mukherjee B: Statistical approaches to studies of gene-gene and geneenvironment interaction; in Timothy CBA, Rebbeck R, Shields Peter G (eds): Molecular Epidemiology: Applications in Cancer and Other Human Diseases. New York: Informa HealthCare, 2008.

3 Kraft P, Hunter DJ: The challenge of assessing complex gene-environment and genegene interactions; in Muin MG, Khoury J, Bradley Linda, Little Julian, Higgins Julian, Ioannidis John PA (eds): Human Genome Epidemiology: Building the Evidence for Using Genetic Information to Improve Health and Prevent Disease. In press, 2008.

-4 Dempfle A, Scherag A, Hein R, Beckmann L, Chang-Claude J, Schafer H: Gene-environment interactions for complex traits: definitions, methodological requirements and challenges. Eur J Hum Genet 2008;16:11641172.

5 Hein R, Beckmann L, Chang-Claude J: Sample size requirements for indirect association studies of gene-environment interactions $(\mathrm{G} \times \mathrm{E})$. Genet Epidemiol 2008;32:235245.

-6 Kraft P, Yen YC, Stram DO, Morrison J, Gauderman WJ: Exploiting gene-environment interaction to detect genetic associations. Hum Hered 2007;63:111-119.

7 Garcia-Closas M, Rothman N, Lubin J: Misclassification in case-control studies of geneenvironment interactions: assessment of bias and sample size. Cancer Epidemiol Biomarkers Prev 1999;8:1043-1050.

8 Garcia-Closas M, Thompson WD, Robins JM: Differential misclassification and the assessment of gene-environment interactions in case-control studies. Am J Epidemiol 1998; 147:426-433.

-9 Wong MY, Day NE, Luan JA, Wareham NJ: Estimation of magnitude in gene-environment interactions in the presence of measurement error. Stat Med 2004;23:987-998.
10 Zhang L, Mukherjee B, Ghosh M, Gruber S, Moreno V: Accounting for error due to misclassification of exposures in case-contro studies of gene-environment interaction. Stat Med 2008;27:2756-2783.

11 Liu X, Fallin MD, Kao WH: Genetic dissection methods: designs used for tests of geneenvironment interaction. Curr Opin Genet Dev 2004; 14:241-245

12 Berrettini W, Yuan X, Tozzi F, Song K, Francks C, Chilcoat H, Waterworth D, Muglia P, Mooser V: Alpha-5/alpha-3 nicotinic receptor subunit alleles increase risk for heavy smoking. Mol Psychiatry 2008; 13: 368-373.

13 Bierut LJ, Stitzel JA, Wang JC, Hinrichs AL, Grucza RA, Xuei X, Saccone NL, Saccone SF, Bertelsen S, Fox L, Horton WJ, Breslau N, Budde J, Cloninger CR, Dick DM, Foroud T, Hatsukami D, Hesselbrock V, Johnson EO, Kramer J, Kuperman S, Madden PA, Mayo K, Nurnberger J Jr, Pomerleau O, Porjesz B, Reyes O, Schuckit M, Swan G, Tischfield JA, Edenberg HJ, Rice JP, Goate AM: Variants in nicotinic receptors and risk for nicotine dependence. Am J Psychiatry 2008;165:11631171.

14 Thorgeirsson TE, Geller F, Sulem P, Rafnar T, Wiste A, Magnusson KP, Manolescu A, Thorleifsson G, Stefansson $H$, Ingason A, Stacey SN, Bergthorsson JT, Thorlacius S, Gudmundsson J, Jonsson T, Jakobsdottir M, Saemundsdottir J, Olafsdottir O, Gudmundsson LJ, Bjornsdottir G, Kristjansson K, Skuladottir H, Isaksson HJ, Gudbjartsson T, Jones GT, Mueller T, Gottsater A, Flex A, Aben KK, de Vegt F, Mulders PF, Isla D, Vidal MJ, Asin L, Saez B, Murillo L, Blondal T, Kolbeinsson H, Stefansson JG, Hansdottir I, Runarsdottir V, Pola R, Lindblad B, van Rij AM, Dieplinger B, Haltmayer M, Mayordomo JI, Kiemeney LA, Matthiasson SE, Oskarsson H, Tyrfingsson T, Gudbjartsson DF, Gulcher JR, Jonsson S, Thorsteinsdottir U, Kong A, Stefansson K: A variant associated with nicotine dependence, lung cancer and peripheral arterial disease. Nature 2008;452: 638-642.
15 Weiss RB, Baker TB, Cannon DS, von Niederhausern A, Dunn DM, Matsunami N, Singh NA, Baird L, Coon H, McMahon WM, Piper ME, Fiore MC, Scholand MB, Connett JE, Kanner RE, Gahring LC, Rogers SW, Hoidal JR, Leppert MF: A candidate gene approach identifies the CHRNA5-A3-B4 region as a risk factor for age-dependent nicotine addiction. PLoS Genet 2008;4: e1000125.

16 Amos CI, Wu X, Broderick P, Gorlov IP, Gu J, Eisen T, Dong Q, Zhang Q, Gu X, Vijayakrishnan J, Sullivan K, Matakidou A, Wang Y, Mills G, Doheny K, Tsai YY, Chen WV, Shete S, Spitz MR, Houlston RS: Genomewide association scan of tag SNPs identifies a susceptibility locus for lung cancer at 15q25.1. Nat Genet 2008;40:616-622.

17 Hung RJ, McKay JD, Gaborieau V, Boffetta P, Hashibe M, Zaridze D, Mukeria A, Szeszenia-Dabrowska N, Lissowska J, Rudnai P, Fabianova E, Mates D, Bencko V, Foretova L, Janout V, Chen C, Goodman G, Field JK, Liloglou T, Xinarianos G, Cassidy A, McLaughlin J, Liu G, Narod S, Krokan HE, Skorpen F, Elvestad MB, Hveem K, Vatten L, Linseisen J, Clavel-Chapelon F, Vineis P, Bueno-de-Mesquita HB, Lund E, Martinez C, Bingham S, Rasmuson T, Hainaut P, Riboli E, Ahrens W, Benhamou S, Lagiou P, Trichopoulos D, Holcatova I, Merletti F, Kjaerheim K, Agudo A, Macfarlane G, Talamini R, Simonato L, Lowry R, Conway DI, Znaor A, Healy C, Zelenika D, Boland A, Delepine M, Foglio M, Lechner D, Matsuda F, Blanche H, Gut I, Heath S, Lathrop M, Brennan P: A susceptibility locus for lung cancer maps to nicotinic acetylcholine receptor subunit genes on 15q25. Nature 2008; 452:633-637.

18 Chanock SJ, Hunter DJ: Genomics: when the smoke clears. Nature 2008;452:537-538.

19 Wacholder S, Chatterjee N, Caporaso N: Intermediacy and gene-environment interaction: the example of CHRNA5-A3 region, smoking, nicotine dependence, and lung cancer. J Natl Cancer Inst 2008;100:14881491. 
-20 Chakraborty J, Zandbergen PA: Children at risk: measuring racial/ethnic disparities in potential exposure to air pollution at school and home. J Epidemiol Community Health 2007;61:1074-1079.

-21 Chaturvedi N: Ethnicity as an epidemiological determinant - crudely racist or crucially important? Int J Epidemiol 2001;30:925927.

-22 Gauderman WJ: Sample size requirements for matched case-control studies of gene-environment interaction. Stat Med 2002;21: 35-50.

23 Rothman KJ, Greenland S: Modern Epidemiology (ed 2). Philadelphia, Lippincott-Raven, 1980, pp 329-341.

24 Longmate JA: Complexity and power in case-control association studies. Am J Hum Genet 2001;68:1229-1237.

-25 Mukherjee B, Ahn J, Gruber SB, Rennert G, Moreno V, Chatterjee N: Tests for gene-environment interaction from case-control data: a novel study of type I error, power and designs. Genet Epidemiol, 2008;32:615-626.
26 Greenland S: The effect of misclassification in the presence of covariates. Am J Epidemiol 1980;112:564-569.

27 Marshall JR, Hastrup JL: Mismeasurement and the resonance of strong confounders: uncorrelated errors. Am J Epidemiol 1996; 143:1069-1078.

28 Nieto-Garcia FJ, Bush TL, Keyl PM: Body mass definitions of obesity: sensitivity and specificity using self-reported weight and height. Epidemiology 1990;1:146-152.

29 Thompson WD: Effect modification and the limits of biological inference from epidemiologic data. J Clin Epidemiol 1991;44:221232.

30 Piegorsch WW, Weinberg CR, Taylor JA: Non-hierarchical logistic models and caseonly designs for assessing susceptibility in population-based case-control studies. Stat Med 1994;13:153-162.

31 Mukherjee B, Chatterjee N: Exploiting geneenvironment independence for analysis of case-control studies: an empirical Bayestype shrinkage estimator to trade-off between bias and efficiency. Biometrics, 2007;64:685-694.
32 Holcroft CA, Spiegelman D: Design of validation studies for estimating the odds ratio of exposure-disease relationships when exposure is misclassified. Biometrics 1999;55: 1193-1201.

33 Kuha J: Corrections for exposure measurement error in logistic regression models with an application to nutritional data. Stat Med 1994;13:1135-1148.

34 Morrissey MJ, Spiegelman D: Matrix methods for estimating odds ratios with misclassified exposure data: extensions and comparisons. Biometrics 1999;55:338-344.

35 Spiegelman D, Carroll RJ, Kipnis V: Efficient regression calibration for logistic regression in main study/internal validation study designs with an imperfect reference instrument. Stat Med 2001;20:139-160.

36 Wong MY, Day NE, Luan JA, Chan KP, Wareham NJ: The detection of gene-environment interaction for continuous traits: should we deal with measurement error by bigger studies or better measurement? Int J Epidemiol 2003;32:51-57. 